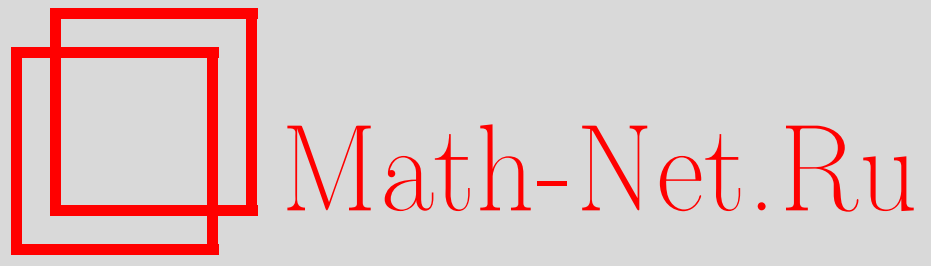

Обшероссийский математический портал

В. Г. Кротов, Когда ортогональный ряд является рядом Фурье, Матем. заметки, 2003, том 74, выпуск 1, 139-142

DOI: https://doi.org/10.4213/mzm586

Использование Общероссийского математического портала Math-Net.Ru подразумевает, что вы прочитали и согласны с пользовательским соглашением http://www. mathnet.ru/rus/agreement

Параметры загрузки:

IP : 3.89 .197 .203

26 апреля 2023 г., 13:15:52 


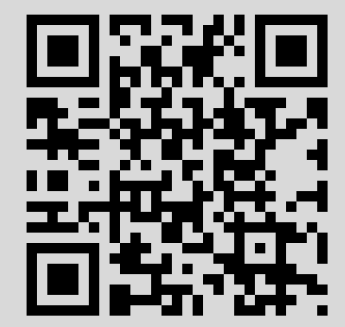




\section{КОГДА ОРТОГОНАЛЬНЫЙ РЯД ЯВЛЯЕТСЯ РЯДОМ ФУРЬЕ}

\section{В.Г. Кротов}

В работе [1] (см. также [2, гл. VI, § 4]) Орлич нашел необходимые и достаточные условия для того, чтобы ряд по ортонормированной системе (ОНС) был рядом Фурье функции из того или иного класса (см. замечание 1 ниже). Эти условия выражаются в терминах регулярных средних ортогонального ряда. Доказательство Орлича проходит, на самом деле, в гораздо более общей ситуации, которую мы сейчас опишем.

Пусть $X$ - банахово пространство надполем скаляров $\mathbb{F}(\mathbb{F}=\mathbb{R}$ или $\mathbb{F}=\mathbb{C}), \Phi=\left\{\phi_{k}\right\}_{k=1}^{\infty} \subset X$ - последовательность элементов из $X, \mathscr{L}_{\Phi}$-линейная оболочка этой системы, $X_{\Phi}-$ замькание $\mathscr{L}_{\Phi}$ по норме $X$. Будем предполагать, что существуют счетное множество $\Phi^{*}=\left\{\phi_{k}^{*}\right\}_{k=1}^{\infty}$ и функция $\langle\cdot, \cdot\rangle: X \times \Phi^{*} \mapsto \mathbb{F}$, линейная и непрерьвная по первой переменной и удовлетворяющая условию

$$
\left\langle\phi_{k}, \phi_{i}^{*}\right\rangle=\delta_{i k}, \quad i, k \in \mathbb{N} \text {. }
$$

Условие (1) позволяет определить ряды Фурье

$$
x \sim \sum_{k=1}^{\infty}\left\langle x, \phi_{k}^{*}\right\rangle \phi_{k}, \quad x \in X .
$$

Будем говорить, что выполнено условие (А), если для любой ограниченной последовательности $\left\{p_{n}\right\} \subset \mathscr{L}_{\Phi}$ существуют $x \in X$ и $n_{j} \uparrow \infty$, для которых

$$
\forall i \in \mathbb{N} \lim _{j \rightarrow \infty}\left\langle p_{n_{j}}, \phi_{i}^{*}\right\rangle=\left\langle x, \phi_{i}^{*}\right\rangle
$$

Условие (А) (представляющее собой рабочую форму условия " $X$ - сопряженное пространство") является, по существу, ядром доказательства приведенного ниже предложения, дающего критерий для свойства "ряд $\sum_{k=1}^{\infty} a_{k} \phi_{k}$ является рядом Фурье" в терминах матричных средних этого ряда.

Пусть $T=\left\{t_{n k}\right\} \subset \mathbb{F}$ - конечнострочная матрица (для каждого $n \in \mathbb{N}$ существует такое $m_{n} \in$ $\mathbb{N}$, что $t_{n k}=0$ при $\left.k>m_{n}\right)$. Ниже будем использовать условие

$$
\forall k \in \mathbb{N} \exists \lim _{n \rightarrow \infty} t_{n k}=t_{k}, \quad \underset{k \rightarrow \infty}{\lim _{k}}\left|t_{k}\right|>0 .
$$

(C) В.Г. Кротов 
Матрица $T$ порождает средние ряда Фурье

$$
T_{n} x=\sum_{k=1}^{m_{n}} t_{n k}\left\langle x, \phi_{k}^{*}\right\rangle \phi_{k}, \quad n \in \mathbb{N} .
$$

Орлич рассматривал следующие формы свойства "ряд $\sum_{k=1}^{\infty} a_{k} \phi_{k}$ является рядом Фурье" ${ }^{1}$ :

$$
\begin{aligned}
& \sum_{k=1}^{\infty} a_{k} \phi_{k}-\text { ряд Фурье элемента из } X \Longleftrightarrow \sup _{n}\left\|\sum_{k=1}^{m_{n}} t_{n k} a_{k} \phi_{k}\right\|_{X}<\infty, \\
& \sum_{k=1}^{\infty} a_{k} \phi_{k}-\text { ряд Фурье элемента из } X \Longleftrightarrow \sum_{k=1}^{m_{n}} t_{n k} a_{k} \phi_{k} \text { сходится по норме } X .
\end{aligned}
$$

В связи с этим естественно рассмотреть условие

$$
\sup _{n}\left\|T_{n}\right\|_{X \rightarrow X}<\infty
$$

которое является необходимым для (3) и (4). Это следует из теоремы Банаха-Штейнгауза.

Для дальнейшего нам понадобится еще следующее понятие. Последовательность $\gamma=\left\{\gamma_{k}\right\} \subset \mathbb{F}$ назьвается мультипликатором для $X$, если для любого $x \in X$ ряд

$$
\sum_{k=1}^{\infty} \gamma_{k}\left\langle x, \phi_{k}^{*}\right\rangle \phi_{k}
$$

является рядом $\Phi$ урье некоторого элемента $x_{\gamma} \in X$. Класс всех мультипликаторов для $X$ обозначаем через $\mathscr{M}(X)$. Если система $\left\{\phi_{k}^{*}\right\}_{k=1}^{\infty}$ полна на $X$ (т.е. из $\left\langle x, \phi_{k}^{*}\right\rangle=0$ для всех $k \in \mathbb{N}$ следует $x=0)$, то $x_{\gamma} \in X$ определяется однозначно и в таком случае мультипликатор $\gamma$ определяет линейный оператор

$$
M_{\gamma} x=x_{\gamma}
$$

ПРЕДЛОЖЕНИЕ. Пусть матрица Т удовлетворяет условию (2), причем

$$
\frac{1}{t}=\left\{\frac{1}{t_{k}}\right\} \in \mathscr{M}(X) .
$$

Тогда

1) если выполнено условие (A), то (5) $\Longleftrightarrow(3)$;

2) если $\Phi$ замкнута в $X\left(\right.$ m.e. $\left.X_{\Phi}=X\right)$, mо $(5) \Longleftrightarrow(4)$.

ДоКАЗАТЕЛЬСТво. То, что из (3) и из (4) следует (5), уже отмечалось.

Импликация "слева направо" в (3) и (4) мгновенно вытекает из условия (5) (и замкнутости $\Phi$ в случае 2)).

Обратно, если последовательность средних

$$
p_{n}=\sum_{k=1}^{m_{n}} t_{n k} a_{k} \phi_{k}
$$

ограничена, то используем условие (А): для некоторого $x \in X$ и любого $i \in \mathbb{N}$

$$
t_{i} a_{i}=\lim _{j \rightarrow \infty} t_{n_{j} i} a_{i}=\lim _{j \rightarrow \infty}\left\langle\sum_{k=1}^{m_{n_{j}}} t_{n_{j} k} a_{k} \phi_{k}, \phi_{i}^{*}\right\rangle=\left\langle x, \phi_{i}^{*}\right\rangle .
$$

В силу (7) $\left\{a_{i}\right\}$ - последовательность коэффициентов Фурье некоторого элемента из $X$. В случае 2) рассуждение такое же с $n_{j}=j$.

Приведем теперь следствие, относящееся к пространствам $L^{p}=L^{p}(S)(S-$ множество с $\sigma$-конечной мерой) и ортогональным (в смысле $L^{2}$ ) рядам.

\footnotetext{
${ }^{1}$ Мы не останавливаемся на других формах критерия из работы Орлича [1] (см. также [2, гл. VI, $\S 4])$. Они также могут быть рассмотрены при наших предположениях относительно матрицы $T$.

${ }^{2}$ Мы считаем $1 / 0=1$.
} 
СледствиЕ 1. Пусть $1 \leqslant p \leqslant \infty u \Phi \subset L^{p} \cap L^{p^{\prime}}$ - ОНС. Тогда если матрица $T$ удовлетворяет условию (2) $u 1 / t \in \mathscr{M}\left(L^{p}\right)$, то

1) $n p u 1<p \leqslant \infty \quad(5)_{L^{p}} \Longleftrightarrow(3)_{L^{p}}$;

2) при $1 \leqslant p<\infty$, если $\Phi$ замкнута в $L^{p}, \quad(5)_{L^{p}} \Longleftrightarrow(4)_{L^{p}}$.

ДоКАЗАТЕЛЬСТво. Надо взять $\phi_{k}^{*}=\phi_{k}$ и

$$
\langle x, y\rangle=\int_{S} x(t) \overline{y(t)} d t
$$

Тогда (1) - условие ортогональнсти, (А) выполнено, так как $L^{p}$ является сопряженным пространством, и ограниченное множество в нем слабо предкомпактно (см. [2, с. 40]).

ЗАмечание 1. Следствие 1 отличается от теоремы Орлича (см. [2, с. 251-252]) тем, что в ней $t_{k}=1$ (и тогда условия $(2)$ и $(7)$ излишни), а вместо $(5)_{L} p$ использовалось условие ограниченности функций Лебега (из которого вытекает $(5)_{L^{p}}$ для всех $X=L^{p}, 1 \leqslant p \leqslant \infty$ ).

ЗАмЕчАНИЕ 2. Условия, при которых справедливы $(3)_{L^{p}}$ и $(4)_{L^{p}}$ для общих ортогональных систем, изучались в [3], но проверка условия $1 / t \in \mathscr{M}\left(L^{p}\right)$ в [3] (см. [3, лемма 3]) ошибочна, так как использует неверное равенство $M_{1 / t}\left(L^{p} \backslash M_{t}\left(L^{p}\right)\right)=L^{2} \backslash L^{p}$ (см. (6)). Поэтому все теоремы этой работы не обоснованы.

Приведем теперь результаты, аналогичные следствию 1 , но для других функциональных пространств. Мы используем ниже в следствии 2 обозначения и терминологию, связанные с пространствами Орлича $L_{\varphi}^{*}$ из [4], с пространствами Харди $H^{p}$ и функций ограниченного среднего колебания $\mathrm{BMO}$ из [5], с пространством функций ограниченной вариации $V$ из [2]. Через $C$ обозначается, как обычно, пространство непрерьвных функций.

СледСтвиЕ 2. Пусть $\Phi \subset L^{2}-$ ОНС и матрица T удовлетворяет условию (2). Тогда

1) если $\varphi-N$-функиия Юнга, $\psi$-дополнительная для $\varphi N$-функиия Юнга, $\Phi \subset L_{\varphi}^{*} \cap$ $L_{\psi}^{*} u 1 / t \in \mathscr{M}\left(L_{\varphi}^{*}\right), m o(5)_{L_{\varphi}^{*}} \Longleftrightarrow(3)_{L_{\varphi}^{*}} ;$

2) если $\Phi \subset H^{\infty} u 1 / t \in \mathscr{M}\left(H^{1}\right), m o(5)_{H^{1}} \Longleftrightarrow(3)_{H^{1}}$;

3) ecлu $\Phi \subset L^{\infty} u 1 / t \in \mathscr{M}(\mathrm{BMO}), m o(5)_{\mathrm{BMO}} \Longleftrightarrow(3)_{\mathrm{BMO}}$;

4) $е с л u \Phi \subset V u 1 / t \in \mathscr{M}(V)$, mo $(5)_{V} \Longleftrightarrow(3)_{V}$;

5) если $\Phi \subset C$ замкнута в $C$ и $1 / t \in \mathscr{M}(C)$, то (5) $C \Longleftrightarrow(4)_{C}$.

Для доказательства надо лишш проверить условие (А) в случаях 1)-4). Оно вытекает из того, что пространства $L_{\varphi}^{*}, H^{1}, \mathrm{BMO}$ и $V$ являются сопряженными пространствами, и слабой компактности единичного шара в сопряженном пространстве (см. [2, с. 40]). Для пространств Орлича см. [4, теорема 14.2 на с. 153$]$, для $H^{1}$ см. [5, с. 275], для ВМО см. [5, с. 245], для $V$ см. [2, с. 29].

ЗАмЕчАНИЕ 3 . Из следствий 1 и 2 в частном случае тригонометрической системы вытекают результаты работ [6]-[9], где рассматривались пространства $L^{p}(\mathbb{T}), L_{\varphi}^{*}$ и ВМО.

Например, условия следствия 1 вытекают из условий [8] и теоремы Марцинкевича о мультипликаторах в $L^{p}(\mathbb{T}), p \in(1, \infty)([10$, с. 346$])$. В [7] ограничения еще более сильные, чем в [8], и для доказательства использовались неравенство Хаусдорфа-Юнга, теорема Карлесона-Ханта и т.д. Результаты работы [9] вытекают из следствий непосредственно. При этом во всех случаях наши ограничения на матрицу $T$ существенно шире. 


\section{СПИСОК ЦИТИРОВАННОЙ ЛИТЕРАТУРЫ}

1. Orlicz W. // Studia Math. 1929. V. 1. P. 1-39. 2. Качмаж С., Штейнгауз Г. Теория ортогональных рядов. М.: ГИФМЛ, 1958. 3. Бруй И. Н. // Матем. заметки. 2002. Т. 71. № 2. С. 182-193. 4. Красносельский М. А., Рутицкий Я. Б. Выпуклые функции и пространства Орлича. М.: ГИФМЛ, 1958. 5. Гарнетт Дж. Ограниченные аналитические функции. М.: Мир, 1984. 6. Bruj I., Schmieder G. // Acta Sci. Math. (Szeged). 1998. V. 64. P. 483-488. 7. Бруй И. Н. // Матем. заметки. 1997. Т. 62. №5. С. 677-686. 8. Бруй И. Н. // Весці АН Беларусі. Сер. физ. -матем. 2000. №1. С. 46-49. 9. Бруй И. Н. // Матем. сб. 2002. Т. 193. № 4. С. 17-36. 10. Зигмунд А. Тригонометрические ряды. Т. 2. М.: Мир, 1965. 